\title{
The Forward EEG Solutions Can be Computed Using Artificial Neural Networks
}

\author{
Mingui Sun*, Member, IEEE, and Robert J. Sclabassi, Member, IEEE
}

\begin{abstract}
Study of electroencenphalogarphy (EEG) is the one of the most utilized methods in both basic brain research and clinical diagnosis of neurological disorders. Recent technological advances in computer and electronic systems have allowed the EEG to be recorded from large electrode arrays. Modeling the brain waves using a head volume conductor model provides an effective method to localize functional generators within the brain. However, the forward solutions to this model, which represent theoretical potentials in response to current sources within the volume conductor, are difficult to compute because of time-consuming numerical procedures utilized in either the boundary element method (BEM) or the finite element method (FEM). This paper presents a novel computational approach using an artificial neural network (ANN) to map two vectors of forward solutions. These two vectors correspond to different head models but with respect to the same current source. The input vector to the ANN is based on the spherical head model, which can be computed efficiently but involves large errors. The output vector from the ANN is based on the spheroidal model, which is more precise, but difficult to compute directly using the traditional means. Our experiments indicate that this ANN approach provides a remarkable improvement over the BEM and FEM methods: 1) the mean-square error of computation was only approximately $0.3 \%$ compared to the exact solution; 2) the online computation was extremely efficient, requiring only 168 floating point operations per channel to compute the forward solution, and 10.2 K-bytes of storage to represent the entire ANN. Using this approach it is possible to perform real-time EEG modeling accurately on personal computers.
\end{abstract}

Index Terms-Artificial neural network, efficient algorithm, efficient computation, EEG, electrostatic theory, forward problem, head geometry, head model, machine learning, partial differential equation, signal processing, source localization.

\section{INTRODUCTION}

I $\mathrm{N}$ recent years, recording high-resolution electroencephalograms (EEG's) from large electrode arrays has become a clear trend in brain research. It provides important insights into the functioning of the brain by revealing the location and sequence of neural activities, and thereby pinpointing the origins of certain neurological disorders, such as epilepsy, sleep disturbances, psychiatric illness, and brain tumors [1].

Manuscript received May 20, 1999; revised March 10, 2000. This work was supported in part by the Whitaker Foundation under the Biomedical Engineering Grant Program, and by the National Institutes of Health (NIH) under Grants NS38494 and NS39047. Asterisk indicates corresponding author.

*M. Sun is with the Laboratory of Computational Neuroscience, Departments of Neurological Surgery and Biomedical Engineering, Suite B-400, PUH, University of Pittsburgh, Pittsburgh, PA 15213 USA (e-mail: mrsun@neuronet.pitt.edu).

R. J. Sclabassi is with the Laboratory of Computational Neuroscience, Departments of Neurological Surgery, Electrical Engineering, and Biomedical Engineering, University of Pittsburgh, Pittsburgh, PA 15213 USA.

Publisher Item Identifier S 0018-9294(00)06414-4.
Several imaging modalities can be used to localize brain functions. These modalities include functional magnetic resonance imaging (fMRI) and positron emission tomography (PET), which are both based on physical changes induced by cerebral blood flow. Although their spatial resolution is more superior than EEG, these modalities have difficulty in localizing fast functional activity because of their low temporal sensitivity. In contrast, the localization of brain functions based on EEG is not constrained by the large time constant associated with blood flow changes. In addition, EEG costs much less than fMRI, PET, and magnetoencephalography (MEG) which is the twin modality of EEG.

The EEG-based source localization technique includes a key component, a forward head volume conductor model (or, in short, a forward model), which mathematically expresses the relationship between a current dipole inside the volume conductor and the corresponding surface potentials. Due to computational considerations, researchers in source localization have been using simple forward models, such as the sphere [2], [3]. In order to model the variations of conductivity values for different structures within the head, the interior of the sphere is often divided into three or four homogeneous shells; namely the scalp, skull, brain, and, optionally, cerebrospinal fluid [4]-[6]. The mathematical expression for the source-potential relationship of the multishell model contains infinite sums of the Legendre functions which are not computationally favorable. To solve this problem we have recently developed an efficient algorithm, implemented by a short C-program, in which the infinite sums are approximated by a closed-form formula [7]. As a result, the required number of floating-point operations are drastically reduced from over 1500 to about 100 .

Although the spherical model is currently widely utilized, it may introduce large errors in modeling the geometry of the head and the highly inhomogeneous conductivity distribution of tissues. To reduce these sources of error a realistic head-shape model can be constructed from computed tomography (CT) or MRI head scan images. The internal structures of the head are represented in different chambers and the model solutions are solved by using the boundary element method (BEM) [8], [9]. Another type of model utilizes the finite element method (FEM) [10], [11] which allows more complex internal geometry and tissue conductivity features to be included, and is more precise. However, this FEM model is computationally expensive. For instance, Haueisen et al. [11] reported an average CPU time of five hours to compute one single forward solution, using the method of successive over-relaxation for a three-dimensional (3-D) finite element model of the head on an IBM RS/6000, Model 580 workstation with 256 Mbytes of RAM. Although the clock rate 


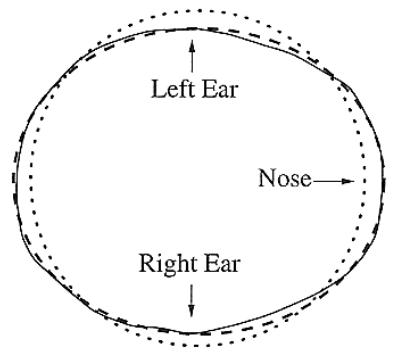

(a)

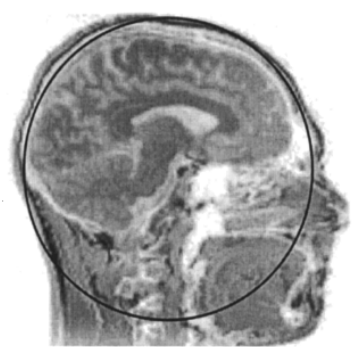

(b)
Fig. 1. (a) Comparison of fitting a head shape (solid curve, representing the top view) by a sphere (dot curve) and a spheroid (dash curve). (b) Sagittal view of the head fit by a circle.

and the size of RAM will both increase as computer technology advances, rapid or real-time EEG modeling based on the traditional approach still does not seem to be feasible in the near future.

A compromise between the modeling error and computational complexity is to model the head as an ellipsoid whose shape is provided by three principal axes. A spheroid is a special case of an ellipsoid in which two of the three axes are equal. The human head is close to a spheroid since the anterior-posterior axis (denoted by $a$ ) is longer than the lateral axis (denoted by $b$ ), while the the sagittal view of the head is essentially circular. Fig. 1(a) compares the realistic head model (solid contour) to the spherical model (dot contour) and the spheroidal model (dash contour). It is clear that the spheroid model provides a good fit.

As in the case of the spherical model, the interior of the spheroidal model can be divided into certain shells with different conductivity values, and the forward solution of this model can be expressed in an analytical form [12]. However, the mathematical expressions of this solution are very complex. Due to the computational complexity involved the spheroidal model has not gained popularity in practice.

In this paper, we present a new artificial neural network (ANN) approach to the computational problem of the spheroidal head model. A function approximation ANN is trained to map the differences between the forward solutions of the spherical and spheroidal models. Once trained, the ANN produces the desired forward solutions of the best-fit spheroid of variable shape. We present the fundamentals of this approach in Section II, the ANN design methods in Section III, and the experimental results in Sections IV and V.

\section{FUNDAMENTALS}

The brain waves observed in EEG data result from synchronous electrical activity of large groups of neurons which can be modeled as electrical current sources. The current generated by these sources flows in closed paths within the electrical conduction media of the head tissues, creating a potential distribution on the scalp. This distribution can be measured noninvasively by electrodes affixed to the skin surface. The volume conduction phenomenon can be described mathematically using the following partial differential equation [13]:

$$
\nabla \cdot(\sigma(\mathbf{r}) \nabla \psi(\mathbf{r}))=s(\mathbf{r}), \quad \text { for } \mathbf{r} \in \Omega
$$

where $\psi(\mathbf{r})$ is the potential function of spatial vector $\mathbf{r}$; $\sigma(\mathbf{r})$ is the conductivity tensor (generally anisotropic); $s(\mathbf{r})$ is the current source density function; $\Omega$ specifies the "boundary condition," and $\nabla$ is the gradient operator, $\nabla f=((\partial f / \partial x),(\partial f / \partial y),(\partial f / \partial z))$, which results in a vector when operating on a scalar, and a scalar when taking a dot-product with a vector.

The EEG waves usually do not originate from a single source. Thus, the forward solution must reflect the nature of multiple sources. It can be easily verified that, if for fixed $\sigma$ and $\Omega$, both

$$
\nabla \cdot\left(\sigma(\mathbf{r}) \nabla \psi_{a}(\mathbf{r})\right)=s_{a}(\mathbf{r})
$$

and

$$
\nabla \cdot\left(\sigma(\mathbf{r}) \nabla \psi_{b}(\mathbf{r})\right)=s_{b}(\mathbf{r})
$$

are true, then

$$
\nabla \cdot\left[\sigma(\mathbf{r}) \nabla\left(\psi_{a}(\mathbf{r})+\psi_{a}(\mathbf{r})\right)\right]=s_{a}(\mathbf{r})+s_{b}(\mathbf{r})
$$

must also be true. This result is usually called the "superposition principle" which states that the forward solutions of multiple sources can be obtained by combining the forward solutions of single elementary sources. The most utilized source model is a current dipole in which the distance between the two poles approaches zero [13].

Although the superposition principle simplifies the forward problem considerably, no analytical forward solutions exist for the realistic head model, even for the simple dipole case. However, we claim that the realistic model solutions are not drastically different from those of the spherical model, as long as the head shape is not deviated greatly from the sphere. The reason is illustrated as follows.

Equation (1) can be considered as an extension of the familiar Ohm's law to the case of volume conduction. Hence, we make use of the Ohm's law ( $V=R I$ ) to show the validity of our claim. Let us consider the following hypothetical processes: 1) constructing a very large, multilayer three-dimensional network of resistors forming a head shape and 2) activating this network by connecting an ideal current source to a single resister branch (in series) inside the network. It is clear that, when the location of the current source is not too close to the outer-most layer of the network, adding or removing a small number of resistors, which is equivalent to a modification of the boundary condition $\Omega$, at the outer-most layer of this network will not cause a large variation of the voltage distribution throughout this network. This observation suggests that $\psi$ is continuous with respect to $\Omega$, i.e., if the variation of $\Delta \Omega$ is small, the variation of $\Delta \phi$ is also small.

Now, let us express this continuity mathematically. Under certain conditions there should exist a continuous function, $f$, given by

$$
\phi_{2}(\Omega+\Delta \Omega, \sigma, s)=f\left(\Delta \Omega, \phi_{1}(\Omega, \sigma, s)\right)
$$

where $\phi_{1}$ and $\phi_{2}$ are, respectively, the forward solutions of a simple base model, such as the sphere, and a deviated model which is closer to the head shape, such as the best-fit spheroid. Although $f$ cannot be expressed explicitly, this difficulty does 
not prevent us from approximating $f$ numerically in an off-line process, since both $\phi_{1}$ and $\phi_{2}$ in (5) can be evaluated based on their respective models, $\Omega$ and $\Omega+\Delta \Omega$, excited by the same current source, $s$. By varying the location and direction of $s$, a large set of samples of the input and output of $f$ can be generated. This forms the basis of our function approximation approach to the forward EEG problem.

The remaining question is how to represent these samples for each head shape. One way to do this is to establish a computer database consisting of many tables indexed by a set of representative head shapes. Once this database is established, future computation can be performed by a table look-up. Although this approach is not impossible with today's computing environment, it is certainly cumbersome requiring tremendous storage. In addition, the potentials stored are highly correlated among different tables, resulting in a very inefficient data representation.

Storing data requires a certain type of "memory". Conventional memory stores values in individual addresses, while associative memory stores contents and relationships in shared addresses [14], [15]. The latter form of memory is more suitable to our case since, although the human head has virtually infinite appearances, they share a common set of features. The technological advances in ANN's have provided various ways to construct the associative memory by performing pattern mapping and functional approximation. It has been shown [16]-[18] that certain ANN's, such as nonlinearly activated backpropagation, radial basis, and generalized regression ANN's can approximate any continuous function to an arbitrarily small error provided that the numbers of training samples and hidden units are sufficiently large. This universal approximation theorem suggests that the desired function $f$ in (5) may be approximated closely by an ANN using machine learning. Once trained the ANN is expect to fetch and generalize the stored information from its associative memory and reproduce consistent output for any head shape which has been, or is related to one of those, observed previously.

\section{Construction of the ANN-BAsed SpheroidAl Model}

The ANN approach presented in the previous section is not restricted to the spheroidal model. However, for realistic head models, generation of training patterns for the ANN requires considerable preprocessing, such as image segmentation, registration, and reconstruction using MRI or CT head scans, surface or volumetric mesh building, and implementation of finite boundary model or FEM. In order to bypass these preprocessing procedures and initiate the study on the ANN approach, we focus on a simple case where the forward solutions of a unit-radius homogeneous spherical model are mapped to that of a spheroidal model of variable shapes. Since both models have closed-form analytical solutions, we do not have to rely on FBM or FEM to obtain forward solutions. In addition, the ANN to be designed is not affected by the numerical errors resulting from FBM or FEM. We anticipate that the design and implementation methods obtained from this simple case will help us in the future investigation on more general cases, where more complex network design and a longer training time may be required.

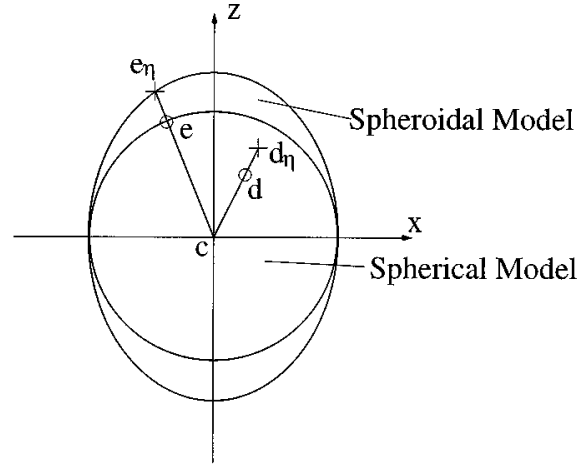

Fig. 2. One-to-one mappings between the electrode sites (e and $\mathbf{e}_{\eta}$ ) and between dipole locations ( $\mathbf{d}$ and $\mathbf{d}_{\eta 7}$ ) for the spherical model (denoted by "o") and the spheroidal model (denoted by "十").

\section{A. Model Specification}

The head model under consideration is a prolate spheroid whose surface equation is given by

$$
\frac{a^{2}\left(x^{2}+y^{2}\right)}{b^{2}}+z^{2}=a^{2}
$$

where $a$ and $b$ are, respectively, the long and short axes. We define the eccentricity $\eta$ by $\eta=\sqrt{1-(b / a)^{2}}$. For most human head shapes, the $\eta$ value is estimated between 0.4 and 0.6 . We also assume, without loss of generality, $b=1$, since the surface potentials on the unit spheroid can always be scaled up to fit different head sizes.

In the ANN approach, we must also choose a base model. For simplicity we employ the simplest homogeneous spherical head model with a unit radius whose equation is given by

$$
x^{2}+y^{2}+z^{2}=1 \text {. }
$$

Equations (6) and (7) imply that the two models share the same coordinate system centered at $c$ as illustrated in Fig. 2.

\section{B. ANN Design}

Once the base (i.e., spherical) and deviated (i.e., spheroidal) models are specified, training patterns for the ANN can be generated by assuming dipole positions and moments and computing forward solutions. The forward solution to (1) for the unit-radius spherical volume conductor with a homogeneous conductivity has the following simple form [19]:

$$
\phi=\frac{1}{4 \pi \sigma q} \sum_{i=1}^{3} m_{i}\left[\frac{2\left(e_{i}-d_{i}\right)}{q^{2}}+e_{i}+\frac{e_{i} s-d_{i}}{q+1-s}\right]
$$

where $d_{i}, m_{i}$, and $e_{i}, i=1,2,3$, are, respectively, the vector elements of $\mathbf{d}, \mathbf{m}$, and e representing the dipole location, current moment, and scalp location; $\sigma$ and $\phi$ are, respectively, the conductivity value and potential at $\mathbf{e}$; and $q$ and $s$ are equal to, respectively, $|\mathbf{e}-\mathbf{d}|$ and $\mathbf{e} \cdot \mathbf{d}$.

The analytical forward solution for the homogeneous spheroidal volume conductor has been reported by Yeh and Martinek [20]. Due to the complexity involved, the mathematical details of this solution will not be presented. The evaluation of this solution involves the following procedures: 1) a coordinate transformation from the Cartesian coordinates to the prolate sphe- 
roidal coordinates; 2) an evaluation of the Hassian matrix of the transformation; 3) evaluations of three types of associated Legendre functions of order zero to infinity; and 4) evaluations of nested infinite sums with respect to the orders of the associated Legendre functions and their derivatives. These functions and derivatives can be expressed using various recursive relations; however, many of these relations are numerically unstable. This problem has been discussed in [21] where stable algorithms have been presented. We utilized 30 terms to approximate each of the nested infinite sums. The accuracy in Legendre function evaluation was checked against the tabulated values in [21]. The entire accuracy of potential computation was examined by setting $\eta$ close to zero and comparing the result obtained from (8).

\section{Pattern Generation}

In order for the ANN to approximate the mapping function in (5), we utilized training patterns constructed from densely located unit-strength random dipoles. For each training pattern, we first generated a large set of three-element vectors for dipole location $\mathbf{d}$ using the uniform probability distribution in the range of $[-0.84,0.84]$, where the value of 0.84 corresponds to the boundary between the brain and scalp shells in the spherical head model. Then, we discarded all vectors in this set whose modulus $|\mathbf{d}|$ was greater than 0.84 . The remaining 12000 dipoles densely covered the "brain region" $(|\mathbf{d}|<0.84)$ within the spherical model.

We associated with each dipole a current moment vector $\mathbf{m}$. Each of the three elements in $\mathbf{m}$ was first generated independently using the zero-mean, unit-variance Gaussian distribution. Then, $\mathbf{m}$ was normalized by re-signing it with $\mathbf{m} /|\mathbf{m}|$. Next, each $(\mathbf{d}, \mathbf{m})$ pair was utilized in (8) to compute a 20-element vector $\vec{\phi}_{1}$ at scalp locations defined by the international $10-20$ system [22].

For each $\vec{\phi}_{1}$ of the spherical model we matched it with a 20-element vector $\vec{\phi}_{2}$ of the spheroidal model of variable shape using the following procedure: 1) generate a random number for eccentricity $\eta$ using the uniform probability distribution in the range between 0.4 and $0.6 ; 2$ ) define a one-to-one mapping between the scalp electrode sites of the two models as shown in Fig. 2 where a ray is projected from the center at $c$, through the electrode site at $\mathbf{e}$ on the spherical model, to the electrode site $\mathbf{e}_{\eta}$ on the spheroidal model (see the Appendix for details of calculation); 3) define a one-to-one mapping (again see Fig. 2 and the Appendix) between dipole locations by projecting from $c$ through $\mathbf{d}$ to $\mathbf{d}_{\eta} ;$ and, 4) plug $\left(\eta, \mathbf{d}_{\eta}, \mathbf{m}, \mathbf{e}_{\eta}\right)$ into the analytical form of the spheroidal model to compute the 20-element potential vector $\vec{\phi}_{2}$.

\section{Training}

A backpropagation (BP) ANN was utilized to approximate $f$ in (5). In our ANN design (see Fig. 3) we utilized a bipolar sigmoid activation function (given by $A(x)=2 /\left(1+e^{-x}\right)-1$ ) and a linear activation function, respectively, at the single hidden layer and the output layer. It has been shown [15] that such a configuration satisfies the universal approximation theorem.

The 21-element input vector, $\mathbf{V}_{\text {in }}$ (a column vector), to the ANN was composed of both the shape parameter, $\eta$, and $\vec{\phi}_{1}$,

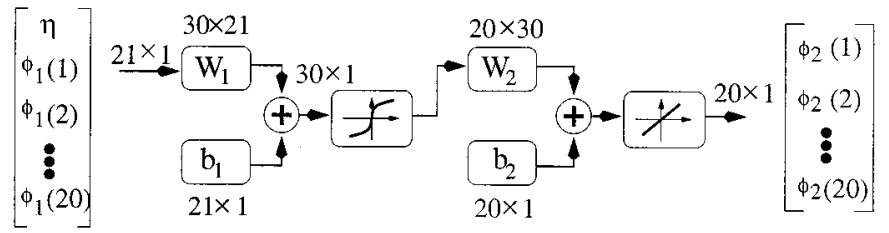

Fig. 3. Configuration of the ANN. $W_{1}$ and $W_{2}$ are weight matrices, and $b_{1}$ and $b_{2}$ are bias vectors. The activation functions (bipolar sigmoid function for the hidden layer, and linear function for the output layer) are illustrated in each box after the add sign $\oplus$. The dimensions of matrices and vectors, as well as the contents of the input/out vectors, are also indicated.

i.e., $\mathbf{V}_{\text {in }}=\left(\eta, \vec{\phi}_{1}\right)^{t}$, while the 20-element output target vector, $\mathbf{V}_{\text {out }}$, consisted of $\vec{\phi}_{2}$ only, i.e., $\mathbf{V}_{\text {out }}=\vec{\phi}_{2}$. Prior to the training process, we normalized both $\mathbf{V}_{\text {in }}$ and $\mathbf{V}_{\text {out }}$ to the unit variance with respect to each row of the 12000 training patterns. This normalization results in an appropriate operating range for each element in the pattern vector, and enables the ANN to be trained more efficiently.

\section{E. Alternative Input/Output Design}

In the previous design $\mathbf{V}_{\text {in }}$ and $\mathbf{V}_{\text {out }}$ are highly correlated because a spheroid with eccentricity $\eta \in[0.4,0.6]$ is not greatly different from a sphere. As a result, we have $\mathbf{V}_{\text {in }}(i+1) \approx$ $\mathbf{V}_{\text {out }}(i)$, for $i=1,2, \ldots, 20$, and the ANN primarily approximates the identical function. In order for the ANN to emphasize the difference between the two models, we re-defined the target training vector as $\mathbf{V}_{\text {out }}^{\prime}(i)=\phi_{2}(i)-\phi_{1}(i)$, for $i=1,2, \ldots, 20$, i.e., $\phi_{1}$ is used to predict $\phi_{2}(i)$. As a result, the identity component is removed from the mapping function, and the average amplitude of $\mathbf{V}_{\text {out }}^{\prime}$ becomes much smaller than $\mathbf{V}_{\text {out }}$. As in the previous case, we normalized both $\mathbf{V}_{\text {in }}$ and $\mathbf{V}_{\text {out }}^{\prime}$ to the unit variance to improve the sensitivity of ANN to the model difference. It is clear that these procedures can be easily reversed to recover $\mathbf{V}_{\text {out }}$ from $\mathbf{V}_{\text {out }}^{\prime}$.

\section{F. Training Algorithms}

There exist numerous training algorithms for the BP ANN. Although some sophisticated designs are involved, no single algorithm is universally better than others. Therefore, the choice of training algorithm is problem-dependent and is usually made by repeated experiments. We investigated five algorithms, including the resilient algorithm [26] and four types of conjugate gradient algorithms [24], [25]. In the resilient algorithm the gradient values in weight update are designed according to the number of consecutive signs in the previous gradient values, rather than their amplitude. In this way the update sensitivity is expected to increase. In the conjugate gradient algorithms, the directions of descent in the optimization process are conjugate each other which, under certain conditions, provide a faster convergence. The four types of conjugate gradient algorithms tested are based on the same principle but differ in the determination of the update constant value (for the Fletcher-Reeves and Polak-Ribiere algorithms), the initialization of a new conjugate set (for the Powell-Beale algorithm), and the method of line search (for the scaled conjugate gradient algorithm). Details of these algorithms are described in [24] and [25]. 
TABLE I

RESUlTS OF ANN PERFoRMANCE Using $\vec{\phi}_{2}$ AS THE TARGET VeCTOR

\begin{tabular}{l|l|l|l|l}
\hline $\begin{array}{l}\text { Training } \\
\text { Method[24, 25] }\end{array}$ & $\begin{array}{l}\text { Training } \\
\text { Time }\end{array}$ & $\begin{array}{l}\text { Epochs } \\
\text { Presented }\end{array}$ & $\begin{array}{l}\text { Relative } \\
\text { Training } \\
\text { Error }\end{array}$ & $\begin{array}{l}\text { Relative } \\
\text { Test Error }\end{array}$ \\
\hline Resilient BP & 573 & 4983 & 0.0039 & 0.0040 \\
Scaled Conjugate & 274 & 1295 & 0.0037 & 0.0039 \\
Fletcher-Reeves & 211 & 1091 & 0.0048 & 0.0051 \\
Polak-Ribiere & 255 & 1286 & 0.0039 & 0.0041 \\
Powell-Beale & 122 & 610 & 0.0043 & 0.0045 \\
\hline
\end{tabular}

\section{EXPERIMENTAL RESULTS}

The BP ANN was implemented on a 9000/802 HP workstation using the Neural Network Toolbox (Version 3) in the Matlab software package (Version 5.2, Mathworks Inc., Natick, MA). Repeated experiments were performed to determine the sizes of hidden neurons and training samples. Our final ANN consists of 30 hidden neurons which provide a compromise between the mapping error and the computational cost. With this design the number of weights is about 1230 . The ratio between the number of training patterns and the number of weights is 10:1, as suggested by a common rule of thumb [23]. Our experiments indicate that more training patterns provide a smaller mapping error and a better generalization; however, a larger dynamic memory (swap space) is required when the batch training, which is more rapid than the sequential training, is employed [24], [25]. We also employed the early stopping criterion in which training was halted when the validation error (computed based on an independent 5000-pattern validation set) started to increase. The results of our experiments are shown in Table I with respect to the training algorithms described previously, training time, number of epochs presented, relative training error, and relative test error. These relative errors are defined by

$$
E=\frac{\sum_{i=1}^{M}\left(\phi_{2}(i)-\hat{\phi}_{2}(i)\right)^{2}}{\sum_{i=1}^{M} \phi_{2}(i)^{2}} \times 100 \%
$$

where $\phi_{2}$ and $\hat{\phi}_{2}$ are, respectively, the directly computed and ANN produced potentials for the spheroidal model, $M=12000$ for the training case, and $M=5000$ for the test case. Note that in the test case the 5000 test patterns were independently generated without being used in the training process.

The modified target vectors were utilized to train another ANN which has the same configuration as in the previous case. The results are listed in Table II. It can be observed that both the training and test relative errors have been improved significantly. In order to visualize the accuracy of the ANN computation more directly, we selected a single test pattern from the 5000-pattern test set and compared, in Fig. 4, the forward solutions computed by the ANN (dashed curve), by direct evaluation (solid curve), and by the spherical head model as the input to the ANN (dotted curve). In this example the ANN was trained using the Powell-Beale algorithm. It can be seen that the solid
TABLE II

RESUlts OF ANN PERFORMANCE USING $\vec{\phi}_{2}-\vec{\phi}_{1}$ AS THE TARGET VeCTOR

\begin{tabular}{l|l|l|l|l}
\hline $\begin{array}{l}\text { Training } \\
\text { Method[24, 25] }\end{array}$ & $\begin{array}{l}\text { Training } \\
\text { Time } \\
\text { (min.) }\end{array}$ & $\begin{array}{l}\text { Epochs } \\
\text { Presented }\end{array}$ & $\begin{array}{l}\text { Relative } \\
\text { Training } \\
\text { Error }\end{array}$ & $\begin{array}{l}\text { Relative } \\
\text { Test Error }\end{array}$ \\
\hline Resilient BP & 200 & 1739 & 0.0029 & 0.0031 \\
Scaled Conjugate & 70 & 330 & 0.0030 & 0.0032 \\
Fletcher-Reeves & 43 & 245 & 0.0031 & 0.0033 \\
Polak-Ribiere & 240 & 1189 & 0.0026 & 0.0028 \\
Powell-Beale & 95 & 453 & 0.0028 & 0.0030 \\
\hline
\end{tabular}

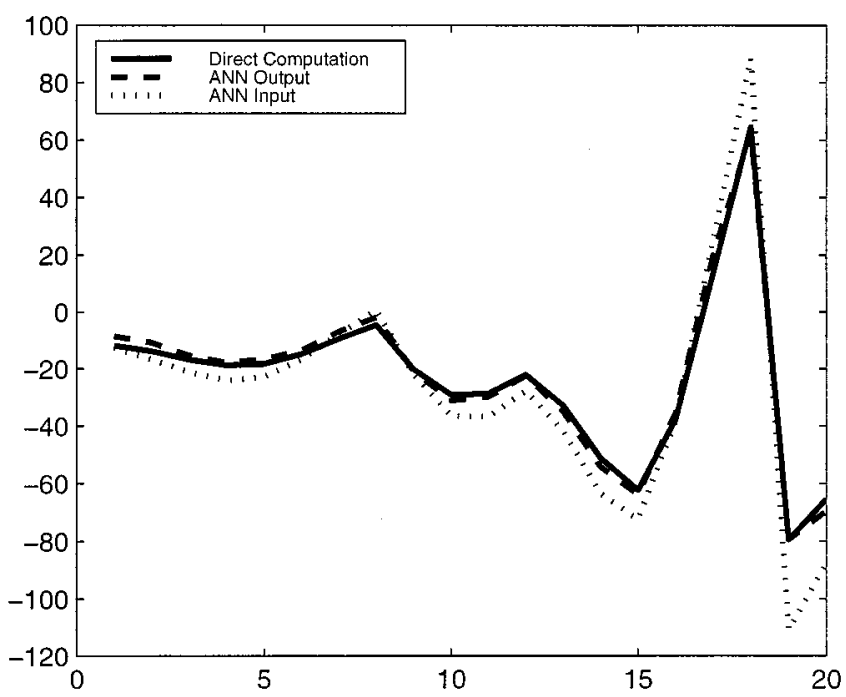

Fig. 4. A particular potential pattern selected from a 5000-pattern test set. The comparison is made among the ANN computed result (dash curve), the directly evaluated result (solid curve), and the result evaluated based on the spherical model which is used as the input to the ANN (dot curve). The horizontal and vertical axes represent, respectively, channel number and potential value (in millivolts). In this comparison the relative mean-squared error is 0.0046 .

and dashed curves are very close, indicating a good approximation by the ANN. Note that most other forward solutions in the 5000-pattern test set performed better than this solution because, in this particular case, the relative test error is 0.0046 , larger than the average relative test error of 0.0030 as listed in the last cell in Table II.

The training times for the ANN listed on Tables I and II are not overwhelming for an off-line, unattended computation. Once the ANN is trained, the on-line computation can be performed very rapidly as shown by the following measure of the number of floating point operations (flops). The total flops required consist of two major components: 1) those required for evaluating (8) which are estimated to be 45 flops per channel, or 900 flops for 20 channels; and 2) those required for evaluating the ANN in the form of multiplications between weight matrices and data vectors, where an $M \times N$ matrix multiplying a $M$-dimensional vector requires $(2 M-1) N$ flops. In our case the ANN has a 21:30:20 configuration. The flops required to evaluate the ANN are approximately $21 \times 2 \times 30+30 \times 2 \times 20=2460$ flops. Therefore, to compute 20-channels of forward solutions, the total computational cost is only $900+2460=3360$ flops. 
If real-time processing is desired, these flops must be accomplished within $5 \mathrm{~ms}$ (assuming a sampling rate $200 \mathrm{~Hz}$ ). This task represents little problem since a $400-\mathrm{MHz}$ PC is capable of accomplishing 100000 flops in 5 ms under a very conservative estimate of 20 clock cycles per flop.

The trained network requires storage for weights and biases. In our case we must store $21 \times 30+30 \times 20=1230$ values for the weights and $30+20=50$ values for the biases (Fig. 3). Assuming each value is stored in the double precision floatingpoint format consisting of 8 bytes, the total storage required is only about $10.2 \mathrm{~K}$-bytes.

\section{DISCUSSION}

We have presented an ANN approach to compute EEG forward solutions for a spheroidal model of variable shape. The computational speed of this new approach out-performs that of the direct computation by a very wide margin, making it possible to use precise head models in practical EEG analysis demanding little computational resources. This approach, with certain modifications, can also be applied to the modeling of MEG, ECG, EMG, various other biomedical data, and nonbiomedical engineering and scientific problems in which efficient computation of numerical solutions to certain partial differential equations is required.

At the current stage we have only tested a simple case where the forward solutions of the homogeneous spherical and spheroidal models were successfully mapped. Further investigations are required for more complex cases where the input and output of the ANN may be designed as follows. The input vector consists of three parts: 1) head shape parameters, 2) conductivity parameters, and 3 ) potentials computed from the multishell spherical model implemented using an efficient algorithm [7]. The output vector contains the potentials corresponding to the FBM or FEM. With this design the ANN is instructed to select a set of forward solutions from its associative memory according to the boundary condition $\Omega$ and conductivity $\sigma$ specified in the first two parts of the input vector. As a result, a single ANN is capable of representing different head shapes and conductivity characteristics. Considering that the forward solutions for different head sizes of the same shape can be obtained by a simple scaling, this ANN may satisfy the needs in EEG modeling for a great portion of the general population.

Although the utilization of ANN to solve the forward problem is promising, there are several issues to be addressed: 1) We do not currently have accurate measures of tissue conductivity (generally anisotropic); 2) The methods for compact representations of head shape and conductivity are not currently available; 3) The input/output potential vectors are not necessarily unique, i.e., one single set of potentials of the base model may have more than one corresponding sets of potentials of the deviated model, or vice versa. This nonuniqueness may affect mapping accuracy when the number of channels is small; 4) As the number of EEG channels increases, the vector size may become excessively large. In this case it may become necessary to represent scalp potentials by compact parameters instead of raw values; 5) A systematic procedure is required to determine electrode locations on the head so that these positions match the "standard" positions from which the ANN was designed. Although considerable effort is required to solve these problems, there have been many years of research in image processing, FEM and BEM optimization, shape description, data parameterization, and data compression. We believe that the ANN approach indeed provides a practical solution to the EEG modeling problem.

\section{APPENDIX \\ MAPPING PoINTS BETWEen THE SPHERICAL AND SPHEROIDAL SPACES}

The mapping between electrode positions $\mathbf{e}=\{\hat{x}, \hat{y}, \hat{z}\}$ and $\mathbf{e}_{\eta}=\{\tilde{x}, \tilde{y}, \tilde{z}\}$ has been illustrated in Fig. 2. The line, denoted by $l$, passes through points $c$ and $\mathbf{e}$. Hence, its line equation is given by

$$
\frac{x}{\sin \psi_{0} \cos \theta_{0}}=\frac{y}{\sin \psi_{0} \sin \theta_{0}}=\frac{z}{\cos \psi_{0}}
$$

where $\theta_{0}$ and $\psi_{0}$ are, respectively, represent the angles to the $x$ and $z$-axes as defined in the spherical coordinate system. By the definition of mapping, line $l$ must also pass $\mathbf{e}_{\eta}$, we then have

$$
\frac{\sin \psi_{1} \cos \theta_{1}}{\sin \psi_{0} \cos \theta_{0}}=\frac{\sin \psi_{1} \sin \theta_{1}}{\sin \psi_{0} \sin \theta_{0}}=\frac{a \cos \psi_{1}}{\cos \psi_{0}}
$$

where $\psi_{1}$ and $\theta_{1}$ are, respectively, the angles representing $\mathbf{e}_{\eta}$. Note that, in (11), we have assumed that the short axis of the spheroid is equal to one (see Section III-C).

Solving (11), we have

$$
\theta_{1}=\theta_{0}
$$

and

$$
\tan \psi_{1}=a \tan \psi_{0}
$$

By the nature of the problem, line $l$ generally passes through the surface of the spheroid twice. Thus, there are two candidates for $\mathbf{e}_{\eta}$. The desired candidate is the one with a smaller $\left|\mathbf{e}-\mathbf{e}_{\eta}\right|$. Using the above results, we can now explicitly express $\mathbf{e}_{\eta}$ by

$$
\begin{aligned}
& \tilde{x}=\sin \psi_{1} \cos \theta_{0} \\
& \tilde{y}=\sin \psi_{1} \sin \theta_{0} \\
& \tilde{z}=a \cos \psi_{1}
\end{aligned}
$$

with

$$
\psi_{1}= \begin{cases}\psi_{0}, & \text { for } \psi_{0}= \pm \frac{\pi}{2} \\ \tan ^{-1}\left(a \tan \psi_{0}\right)+k \pi, & \text { otherwise }\end{cases}
$$

where $k \in\{0,1\}$. The case for mapping the two dipole positions $\mathbf{d}$ and $\mathbf{d}_{\eta}$ (see Fig. 2) can be similarly derived. The results are the same as shown in (13) and (14) except that $\tilde{x}, \tilde{y}$, and $\tilde{z}$ are all multiplied by the modulus $|\mathbf{d}|$.

\section{REFERENCES}

[1] W. W. Orrison, J. D. Lewine, J. A. Sanders, and M. F. Hartshorne, Functional Brain Imaging. St. Louis, MO: Mosby-Year Book, 1995.

[2] F. N. Wilson and R. H. Bayley, "The electrical field of an eccentric dipole in a homogeneous spherical conducting medium," Circulation, vol. 1, pp. 84-92, 1950.

[3] D. A. Brody and W. E. Romans, "A model which demonstrates the quantitative relationship between the electromotive forces of the heart and the extremity leads," Amer. Heart J., vol. 45, pp. 253-266, 1953. 
[4] S. Rush and D. A. Driscoll, "EEG electrode sensitivity-An application of reciprocity," IEEE Trans. Biomed. Eng., vol. BME-16, pp. 15-22, Jan. 1969.

[5] B. N. Cuffin and D. Cohen, "Comparison of the magnetoencephalogram and electroencephalogram," Electroencephalogr. Clin. Neurophysiol., vol. 47, pp. 132-146, 1979.

[6] C. J. Stok, The inverse Problem in EEG and MEG with Application to Visual Evoked Responses. The Hague: CIP Gegevens Koninklijke Bibliotheek, 1986.

[7] M. Sun, "An efficient algorithm for computing multishell spherical head models for EEG source localization," IEEE Trans. Biomed. Eng., vol. 44, pp. 1243-1252, Dec. 1997

[8] B. N. Cuffin, "A method for localizing EEG sources in realistic head models," IEEE Trans. Biomed. Eng., vol. 42, pp. 68-71, Jan. 1995

[9] S. Homma, T. Musha, Y. Nakajima, Y. Okamoto, S. Blom, R. Flink, and K. E. Hagbarth, "Conductivity ratios of the scalp-skull-brain head model in estimation equivalent dipole sources in human brain," Neurosci. Res., vol. 22, pp. 51-55, 1995.

[10] Numerical Methods for Bioelectric Field Problems, Biomedical Engineering Handbook, J. D. Bronzino, Ed., CRC, Boca Raton, FL, 1985, pp. $161-188$.

[11] J. Haueisen, C. Ramon, M. Eiselt, and B. B. Hartmut, "Influence of tissue resistivities on neuromagnetic fields and electric potentials studied with a finite element model of the head," IEEE Trans. Biomed. Eng., vol. 44, pp. 727-735, Aug. 1997.

[12] J. C. de Munck, "The potential distribution in a layered anisotropic spheroidal volume conductor," J. Appl. Phys., vol. 64, pp. 464-470, 1988.

[13] M. Hamalainen, R. Hari, R. J. Ilmoniemi, J. Knuutila, and O. V. Lounasmaa, "Magnetoencephalography-Theory, instrumentation, and applications to noninvasive studies of the working human brain," Rev. Modern Phys., vol. 65, pp. 413-497, 1993.

[14] M. H. Hassoun, Ed., Associative Neural Memories. New York: Oxford Univ. Press, 1993.

[15] L. Fausett, Fundamentals of Neural Networks. Englewood Cliffs, NJ: Prentice-Hall, 1994.

[16] G. Cybenko, "Approximation by superpositions of a sigmoidal function," Mathematics Contr., Signals, Syst., vol. 2, pp. 303-314, 1989.

[17] K. Funahashi, "On the approximate realization of continuous mappings by neural networks," Neural Networks, vol. 2, pp. 183-192, 1989.

[18] S. Haykin, Neural Networks: A Comprehensive Foundation, Toronto, Canada: Maxwell Macmillan, 1994.

[19] R. D. Sidman, V. Giambalvo, T. Allison, and P. Bergey, "A method for localization of sources of human cerebral potentials evoked by sensory stimuli," Sensory Processes, vol. 2, pp. 116-129, 1978.

[20] G. C. K. Yeh and J. Martinek, "The potential of a general dipole in a homogeneous conducting spheroid," Ann. New York Acad. Sci., vol. 65, pp. 1003-1006, 1956.

[21] "Tables of Associated Legendre Functions," Columbia Univ. Press, New York, Mathematical Tables Project, 1945. Conducted under the sponsorship of the National Bureau of Standard.
[22] K. B. E. Bocker, J. A. G. van Avermaete, and M. M. C. van den BergLenssen, "The international 10-20 system revised: Cartesian and spherical coordinates," Brain Topogr., vol. 6, pp. 231-235, 1994.

[23] K. Swingler, Applying Neural Networks. San Diego, CA: Academic, 1996.

[24] “Neural Network Toolbox," Mathworks, Inc., Natick, MA, User's Guide Mannual, Version 3, 1998

[25] M. T. Hagan, H. B. Demuth, and M. H. Beale, Neural Network Design. Boston, MA: PWS, 1996.

[26] M. Riedmiller and H. Braun, "A direct adaptive method for faster backpropagation learning: The RPROP algorithm," in Proc. IEEE Int. Conf. Neural Networks, 1993, pp. 586-591.

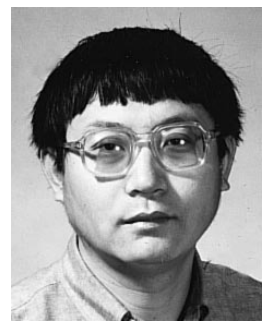

Mingui Sun (S'88-M'89) received the B.S. degree from the Shenyang Chemical Engineering Institute, China, in 1982, and the M.S. and Ph.D. degrees in electrical engineering from the University of Pittsburgh, Pittsburgh, PA, in 1986 and 1989, respectively.

He held various positions in mechanical, electrical, and electronics engineering from 1971 to 1978 with the Chifeng Radio Elements, Inc., China, prior to undergraduate study. He was a Graduate Student Researcher from 1985 to 1989 working on signal and image processing projects. Currently, he is a Associate Professor and an Associate Director of the Center for Clinical Neurophysiology in the Department of Neurosurgery at the University of Pittsburgh, Pittsburgh, PA. His current research interests include advanced biomedical devices, biomedical signal and image processing, artifical neural networks, wavelet transforms, time-frequency analysis, and the inverse problem of neurophysiological signals. He has over 100 publications in these areas.

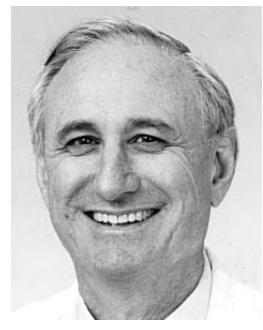

Robert J. Sclabassi (M'62-S'68-M'73-SM'92) received the B.S.E. degree from Loyola University, Los Angeles, CA, the M.S.E.E., Engr's, and Ph.D. degrees in electrical engineering from the University of Southern California, Los Angeles, and the M.D. degree from the University of Pittsburgh, Pittsburgh, PA.

He was employed in the Advanced Systems Laboratory at TRW, Los Angeles, CA, and was a postdoctoral fellow at the Brain Research Institute at the University of California, Los Angeles. He is currently a Professor of Neurological Surgery, Psychiatry, Neuroscience, Electrical Engineering, Mechanical Engineering, and Biomedical Engineering at the University of Pittsburgh.

Dr. Sclabassi is a Registered Professional Engineer. 